\title{
Haar Wavelet Quasilinearization Approach for Solving Nonlinear Boundary Value Problems
}

\author{
Harpreet Kaur ${ }^{1}$, R. C. Mittal ${ }^{2}$, Vinod Mishra ${ }^{3}$ \\ ${ }^{1,3}$ Department of Mathematics, Sant Longowal Institute of Engineering \& Technology, Longowal, India \\ ${ }^{2}$ Department of Mathematics, Indian Institute of Technology Roorkee, Roorkee, India \\ E-mail: \{maanh57,mishravinod560\}@gmail.com,mittalrc@iitr.ernet.in \\ Received May 16, 2011; revised June 14, 2011; accepted June 26, 2011
}

\begin{abstract}
Objective of our paper is to present the Haar wavelet based solutions of boundary value problems by Haar collocation method and utilizing Quasilinearization technique to resolve quadratic nonlinearity in $y$. More accurate solutions are obtained by wavelet decomposition in the form of a multiresolution analysis of the function which represents solution of boundary value problems. Through this analysis, solutions are found on the coarse grid points and refined towards higher accuracy by increasing the level of the Haar wavelets. A distinctive feature of the proposed method is its simplicity and applicability for a variety of boundary conditions. Numerical tests are performed to check the applicability and efficiency. $\mathrm{C}++$ program is developed to find the wavelet solution.
\end{abstract}

Keywords: Haar Wavelets, Quasilinearization Technique, Haar Collocation Method, Boundary Value Problems

\section{Introduction}

Wavelets are mathematical tools that cut up data, functions or operators into different frequency components and then study each component with a resolution matching its scale. Much of the work on Haar functions was performed in the 1930s. In 1909, Haar discovered the simplest function now called as Haar wavelet. The integral of Haar family called Haar operational matrix was derived by Chen and Hsiao [1] in 1997. Since then the solutions of dynamical systems in a wavelet framework took tremendous growth. In order to take the advantages of the local property, many authors researched the Haar wavelet to solve the linear stiff systems and differential equations [2-4].

Haar function is a rectangular pulse pair. It is infact the Daubechies wavelet of order one and is the simplest of the orthonormal wavelets with compact support. As shortcoming, Haar wavelets are not continuous; their derivatives do not exist at the points of discontinuities. Thereby direct application of Haar wavelet is not possible in solving differential equations but here one possibility is through integration of wavelets [2]. The proposed technique in this paper is based on collocation framework and utilizes the capabilities of Haar wavelet basis which permits to enlarge the class of functions through Quasilinearization. Our main concentration on the following type of nonlinear boundary value problems defined in the interval $[a, b]$.

$$
y^{n}(t)=f\left(t, y^{\prime}, y^{\prime \prime}, \cdots \cdots, y^{(n-1)}\right)
$$

subject to boundary conditions

$$
\begin{aligned}
& y(a)=\alpha_{1}, y^{\prime}(a)=\alpha_{2}, \\
& y^{\prime \prime}(a)=\alpha_{3}, \cdots \cdots, y^{(n-1)}(a)=\alpha_{n} \\
& y(b)=\beta_{1}, y^{\prime}(b)=\beta_{2}, \\
& y^{\prime \prime}(b)=\beta_{3}, \cdots \cdots, y^{(n-1)}(b)=\beta_{n}
\end{aligned}
$$

$f$ is a continuous function, in case of nonlinearity of $f$ is at most quadratic in $y$.

\section{Preliminary Works}

\subsection{Haar Wavelets}

Fourier transform analyzes the composition of a given function in terms of sinusoidal waves of different frequencies and amplitudes whereas wavelets analysis tells how a given function changes from one time period to 
the next. Wavelet analysis is also more flexible in sense that one can choose a specific wavelet to match the type of function being analyzed.

For a function $\psi(\cdot)$, defined over the real axis $(-\infty$, $\infty)$, is classed as a wavelet if it satisfies the following three properties:

1) The integral of $\psi(\cdot)$ is zero: $\int_{-\infty}^{\infty} \psi(t) \mathrm{d} t=0$

2) The integral of the square of $\psi(\cdot)$ is unity: $\int_{-\infty}^{\infty} \psi^{2}(t) \mathrm{d} t$ is finite.

3) Admissibility condition:

$$
C_{\psi}=\int_{0}^{\infty} \frac{|\hat{\psi}(\omega)|}{\omega} \mathrm{d} \omega \quad \text { satisfies } 0<C_{\psi}<\infty .
$$

The Haar scaling function for $t \in[0,1]$ is defined as

$$
h_{0}(t)= \begin{cases}1 & \text { if } 0 \leq t \leq 1 \\ 0 & \text { elsewhere }\end{cases}
$$

and corresponding wavelet function

$$
h_{1}(t)= \begin{cases}1 & \text { if } 0 \leq t \leq \frac{1}{2} \\ -1 & \text { if } 0 \leq t \leq \frac{1}{2} \\ 0 & \text { elsewhere }\end{cases}
$$

Also the graph of is shown in Figure 1 [1].

The orthogonality property puts a strong limitation on the construction of wavelets. It is known that the Haar wavelet is the only real valued wavelet that is compactly supported, symmetric and orthogonal. Thus Haar wavelets $h_{i}(t)$ is orthogonal square waves family with magnitude \pm 1 and zero, generally written as

$$
h_{i}(t)=h_{1}\left(2^{j} t-\frac{k}{2^{j}}\right)
$$

for $i \geq 2, i=2^{j}+k+1, j \geq 0,0 \leq k \leq 2^{j}-1$.

\subsection{Multiresolution Analysis}

Objective of this section is to construct a wavelet system, which is complete orthonormal set in $L^{2}(R)$ The idea of multiresolution analysis is to represent a function $f$ as

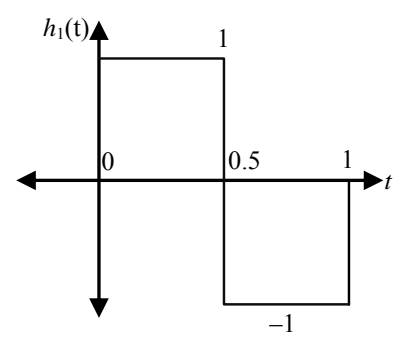

Figure1. First haar wavelet. a limit of successive approximations and decomposition of the whole function space into individual subspaces $V_{j} \subset V_{j+1}$. A multiresolution analysis (MRA) of $L^{2}(R)$ is defined as a sequence of closed subspaces of $V_{j}$ of $L^{2}(R), j \in Z$, that satisfy the following axioms:

1) Monotonicity $\cdots \cdots \cdots \subset V_{-1} \subset V_{0} \subset V_{1} \subset \cdots \cdots \cdots$

2) The spaces $V_{j}$ satisfy $\bigcup_{j \in Z} V_{j}=L^{2}(R)$ and $\bigcap_{j \in Z} V_{j}=$ $\{0\}$.

3) $f(t) \in V_{0}$ iff $f\left(2^{j} t\right) \in V_{j} \forall j \in Z$ i.e. the space $V_{j}$ are scaled versions of the central space $V_{0}$.

4) There exists $\varphi \in V_{0}$ s.t. $\{\varphi(t-k), k \in Z\}$ is a Riesz basis in $V_{0}$.

The sequence $\left\{\varphi_{j, k}(t)=2^{j / 2} \varphi\left(2^{j} t-k\right)\right\}^{\infty}$ forms an orthogonal basis for $V_{j}$ by using the multiresolution analysis axioms. The space $V_{j}$ is used to approximate general functions by defining appropriate projection of these functions onto these spaces.

The vector space $W_{j}$ is the orthogonal complement of $V_{j} \cap V_{j+1}$. In other words, we will let $W_{j}$ be the space of all functions in $V_{j}$ under the chosen inner product. See [5] for detail of MRA. As an example the space $V_{j}$ can be defined like

$$
\begin{aligned}
V_{j} & =W_{j-1} \oplus V_{j-1}=W_{j-1} \oplus W_{j-2} \oplus V_{j-2} \oplus \ldots \ldots \\
& =\oplus_{j=1}^{J+1} W_{j} \oplus V_{0},
\end{aligned}
$$

then the scaling function $h_{1}(t)$ generates an MRA for the sequence of spaces $\left\{V_{j}, j \in Z\right\}$ by translation and dilation as defined in (2) and (3). The linearly independent functions $\psi_{j, k}(t)$ spanning $W_{j}$ are called wavelets. Original signal can be expressed as a linear combination of the box basis functions in $V_{j}$. These basis functions have two important properties: orthogonality property with $\varphi_{0,0}(t), \psi_{0,0}(t), \mid \psi_{1,0}(t), \psi_{1,1}(t)$ and normalization of $\psi_{j, k}(t)=2^{j / 2} \psi\left(2^{j} t-k\right), j, k \in Z$.

\section{Haar Wavelet Integration and the Quasilinearization Approach}

The wavelets corresponding to the box basis are known as the Haar wavelets.

$$
h_{i}(t)= \begin{cases}1 & \text { for } t \in[\alpha, \beta], \\ -1 & \text { for } t \in[\beta, \gamma], \\ 0 & \text { elsewhere }\end{cases}
$$

Here $\alpha=\frac{k}{m}, \beta=\frac{k+0.5}{m}, \gamma=\frac{k+1}{m}, m=2^{j}, \quad j=0,1, \cdots, J$. $J$ indicates the level of resolution. The integer $k=0,1, \cdots, m-1$ is the translation parameter. The indexing $i$ in (4) is calculated as $i=m+k+1$. In case with 
minimal values $m=1, k=0, i=2$. The maximal value of $i$ is $2 m=2^{j+1}$.

Consider the collocation points $t_{l}=\frac{l-0.5}{2 m}$,

$l=1,2, \cdots, 2 m$. The operational matrix $P$ which is a $2 m \times 2 m$ square matrix is defined by

$$
P_{i, 1}(t)=\int_{0}^{t} h_{i}(x) \mathrm{d} x
$$

Remarkable that [3] considers integral of Haar wavelets as

$$
\int_{0}^{t} h_{2 m}(x) \mathrm{d} x \cong P_{2 m \times 2 m} h_{2 m}(t), \quad t \in[0,1)
$$

and operational matrix is

$$
P_{2 m \times 2 m}=\frac{1}{4 m}\left[\begin{array}{ll}
4 m P_{(m) \times(m)} & -H_{(m) \times(m)} \\
H_{(m) \times(m)}^{-1} & 0_{(m) \times(m)}
\end{array}\right],
$$

But we have considered the integral of Haar wavelets as following:

$$
\begin{gathered}
P_{i, 1}(t)=\int_{0}^{t} h_{i}(x) \mathrm{d} x= \begin{cases}t-\alpha & \text { for } t \in[\alpha, \beta] \\
\gamma-t & \text { for } t \in[\beta, \gamma] \\
0 & \text { elsewhere }\end{cases} \\
P_{i, 2}(t)=\left\{\begin{array}{lll}
\frac{1}{2}(t-\alpha)^{2} & \text { for } & t \in[\alpha, \beta] \\
\frac{1}{4 m^{2}}-\frac{1}{2}(t-\alpha)^{2} & \text { for } & t \in[\beta, \gamma] \\
\frac{1}{4 m^{2}} & \text { for } & t \in[\gamma, 1] \\
0 & \text { elsewhere }
\end{array}\right.
\end{gathered}
$$

We also introduce the following notation for specific value of function

$$
D_{i, 1}=\int_{0}^{1} P_{i, 1}(t) \mathrm{d} t
$$

The Quasilinearization technique [6] is an application of the Newton-Raphson-Kantrovich approximation method in function space. This method is applied to solve a nonlinear $n^{\text {th }}$ order ordinary or partial differential equation in N-dimensions as a limit of a sequence of linear differential equations. The idea of the method is based on the fact that how to solve the nonlinear ODE's by Haar wavelets while there are no useful techniques for obtaining the general solution of a nonlinear equation in terms of a finite set of particular solutions. But we limit ourselves here for variables according to involved variables in nonlinear ordinary differential in the interval $[a, b]$, $a=0, b=1$.

$$
L^{(n)} y(t)=f\left(y(t), y^{(1)}(t), y^{(2)}(t), y^{(3)}(t), \cdots, y^{(n-1)}(t), t\right)
$$

with initial conditions

$$
\begin{aligned}
& h\left(y(a), y^{(1)}(a), y^{(2)}(a), \cdots \cdots, y^{(n-1)}(a)\right)=0 \\
& g\left(y(b), y^{(1)}(b), y^{(2)}(b), \cdots \cdots, y^{(n-1)}(b)\right)=0
\end{aligned}
$$

Here $L^{(n)}$ is the linear $n^{\text {th }}$ order ordinary differential operator, $f$ is nonlinear functions of $y(t)$ and its $n-1$ derivatives are $y^{(j)}(t), j=1,2, \cdots \cdots, n-1$.

The Quasilinearization prescription determines the $(r$ $+1)^{\text {th }}$ iterative approximation $y_{r+1}(t)$ to the solution of $n^{\text {th }}$ order nonlinear ordinary differential Equations (1) as a solution of linear differential equation.

$$
\begin{aligned}
& L^{(n)} y_{r+1}(t) \\
= & f\left(y_{r}(t), y_{r}^{(1)}(t), y_{r}^{(2)}(t), \cdots \cdots, y_{r}^{(n-1)}(t), t\right) \\
+ & \sum_{j=0}^{n-1}\left(y_{r+1}^{(j)}(t)-\left(y_{r}^{(j)}(t)\right)\right) \\
& f_{y^{(j)}}\left(y_{r}(t), y_{r}^{(1)}(t), \cdots \cdots, y_{r}^{(n-1)}(t), t\right),
\end{aligned}
$$

where $y_{r}^{0}(t)=y_{r}(t)$. The functions $f_{y^{(j)}}=\frac{\partial f}{\partial y^{j}}$ are functional derivatives of the functional

$$
f\left(y(t), y^{(1)}(t), y^{(2)}(t), \cdots \cdots, y^{(n-1)}(t), t\right)=0
$$

and

$$
\begin{aligned}
& h\left(y(a), y^{(1)}(a), y^{(2)}(a), \cdots \cdots, y^{(n-1)}(a)\right)=0 \\
& g\left(y(b), y^{(1)}(b), y^{(2)}(b), \cdots \cdots, y^{(n-1)}(b)\right)=0 \\
& \delta y_{r+1}(t)=y_{r+1}(t)-y_{r}(t)
\end{aligned}
$$

The zero ${ }^{\text {th }}$ iteration $y_{0}(t)$ is chosen as Haar wavelet basis from physical and mathematical considerations of given problem.

In [7] proved $\left\|\Delta y_{r+1}(t)\right\| \leq k\left\|\Delta y_{r}(t)\right\|^{2}$ that showed the difference between exact solution and the $r^{\text {th }}$ iteration is decreasing quadratically and $\delta y_{r+1}(t)$ for an arbitrary $l<r$ satisfied the following inequality:

$$
\left\|\delta y_{r+1}(t)\right\| \leq\left(k\left\|\delta y_{l}(t)\right\|\right)^{2^{r-1}} / k
$$

the Haar wavelet series considered as function approximation in Quasilinearization technique which satisfies the boundary conditions of given problem. Then Quasilinearization procedure is adopted through Haar wavelet collocation method for getting the solution of concerning problems.

\section{Error Analysis of Haar Wavelets}

Let $\phi$ and $\psi$ are the scaling and the corresponding 
wavelet function of $L^{2}(R)$ respectively, also imply the relations

$$
\int_{-\infty}^{\infty} \varphi(t) \mathrm{d} t=1, \int_{-\infty}^{\infty} \psi(t) \mathrm{d} t=0 \quad \text { (the moment properties). }
$$

The following dilation relation holds:

$$
\varphi(t)=\sqrt{2} \sum_{l=L_{1}}^{L_{2}} h_{l} \varphi(2 t-l)
$$

with some $h l \in R, L_{1}, L_{2} \in Z$ and the family wavelets

$$
\begin{aligned}
& \varphi_{j, k}(t)=2^{\frac{j}{2}} \varphi\left(2^{j} t-k\right), \\
& \psi_{j, k}(t)=2^{\frac{j}{2}} \psi\left(2^{j} t-k\right), \quad j, k \in Z
\end{aligned}
$$

$P_{j}$ and $Q_{j}$ be the corresponding projections

$$
\begin{aligned}
& V_{j+1}=V_{j} \oplus W_{j} \\
& P_{j} y=\sum c_{j, k} \phi_{j, k}, \quad c_{j, k}=\int y \phi_{j, k} \mathrm{~d} t \\
& Q_{j} y=\sum d_{j, k} \psi_{j, k}, \quad d_{j, k}=\int y \psi_{j, k} \mathrm{~d} t
\end{aligned}
$$

Let $M=M_{2}-M_{1} \geq p$ of the supports of wavelets $\varphi$ and $\psi . M$ stands for $M^{\text {th }}$ moment of wavelet function. Then according to [8], we have the following theorem:

Theorem. Assume the moment condition. For $y \in$ $C^{l}(R), \quad 1 \leq k \leq \lambda$, the following estimation holds:

$$
\left|y-P_{j} y\right| \leq A \max _{|w-t| \leq 2^{-j} M}\left|y^{l}(w)\right|, j \in Z
$$

where $y^{l}(w)$ stands for derivative of $y$.

Remark. For Haar function, $M=1,\left(M_{1}=0, M_{2}=1\right)$. Let $y \in L^{2}(R)$ be bounded first derivative on $(0,1)$ such that $\left|y^{l}(w)\right| \leq S$ Then error at $j^{\text {th }}$ level will be given by

$$
\left|y-P_{j} y\right| \leq R S 2^{-k j} \Rightarrow\left|y-P_{j} y\right| \leq A 2^{-k j}
$$

$A$ and $R$ are suitable real constants.

\section{Function Approximations}

Orthogonality of Haar wavelets ensures that any square integral function over $[0,1]$ can be expressed as an infinite sum of Haar wavelets as

$$
f(t)=\sum_{i=1}^{\infty} a_{i} h_{i}(t)
$$

where $a_{i}$ 's are wavelet coefficients.

If $f(t)$ is piecewise constant or can be approximated as piecewise constant during each subinterval, then sum can be terminated to finite term as [8]

$$
y(t)=\sum_{i=1}^{j^{j+1}} a_{i} h_{i}(t)=a^{\mathrm{T}} H
$$

A function $f \in L^{2}(R)$ is a MRA of $L^{2}(R)$ produces a sequence of subspaces $V_{j}$ of $L^{2}(R), j \in Z$ s.t. the projection of $f$ onto these spaces give finer approximation of the function $f$ as $j \rightarrow \infty$.

To demonstrate the applicability of Haar wavelets, we focus on the following nonlinear BVP's and utilizing $\mathrm{C}++$ Programming and MATLAB Software. In finite element method the approximate solution can be written as a linear combination of basis functions which constitute a basis for the approximation space under consideration.

Here in Haar collocation method the series is taken as the highest derivative of given differential equation as a linear combination of Haar wavelet basis.

$$
y^{(n)}(t)=\sum_{i=1}^{2^{j+1}} a_{i} h_{i}(t)
$$

Subsequent integrations give lower derivatives and $y(t)$. Substituting the values in the given equation gives the coefficients and hence the solution.

\section{Test Problems}

\subsection{Nonlinear Boundary Value Problems}

\subsubsection{Two Point Boundary Value Problem}

Firstly, the application of Haar wavelets by Quasilinearization has been performed on the second order BVP of purely mathematical nature [9] which posses analytic solution and given by:

$$
\begin{aligned}
& y^{\prime \prime}=y^{2}(t)+2 \pi^{2} \cos (2 \pi t)-\sin ^{4}(\pi t), \\
& 0 \leq t \leq 1, \quad y(0)=0 \text { and } y(1)=0 .
\end{aligned}
$$

with analytic solution $y(t)=\sin ^{2}(\pi t)$. Approximate the solution as $y_{n}=\sin (\pi t)$

By wavelet based Quasilinearization technique, $y^{2}(t)=2 \sin (\pi t) y(t)-\sin ^{2}(\pi t)$ and Equation (8) becomes

$$
\begin{aligned}
& a\left[H-2 \sin (\pi t) P_{2}+2 t \sin (\pi t) P_{1}\right] \\
& =-\sin ^{2}(\pi t)+2 \pi^{2} \cos (2 \pi t)-\sin ^{4}(\pi t) .
\end{aligned}
$$

Efficiency of method for solution of second order BVP problem is depicted in Figure 2, for $j=2$ and $j=3$.

\subsubsection{Fourth Order Boundary Value Problem}

Consider the fourth order nonlinear boundary value problem [10]

$$
\begin{aligned}
& y^{\prime \prime \prime}=y^{2}-t^{10}+4 t^{9}-4 t^{8}-4 t^{7}+8 t^{6}-4 t^{4}+120 t-48, \\
& 0<t<1 .
\end{aligned}
$$


subject to $y(0)=y^{\prime}(0)=0, y(1)=y^{\prime}(1)=1$.

Approximate the highest derivative as Haar wavelet series $y^{\prime \prime \prime}(t)=\sum_{i=1}^{2^{j+1}} a_{i} h_{i}(t)=a^{\mathrm{T}} H$

$$
\begin{aligned}
& P_{i, 2}(t)=\int_{0}^{t} \int_{0}^{t} h_{i}(t) \mathrm{d} t^{2}, \\
& P_{i, 3}(t)=\int_{0}^{t} \int_{0}^{t} \int_{0}^{t} h_{i}(t) \mathrm{d} t^{3},
\end{aligned}
$$

Solution in first iteration is shown in the Figure 3.

\subsection{Linear Boundary Value Problem}

\section{Fourth Order Linear Boundary Value Problem}

Consider the fourth order linear boundary value problem

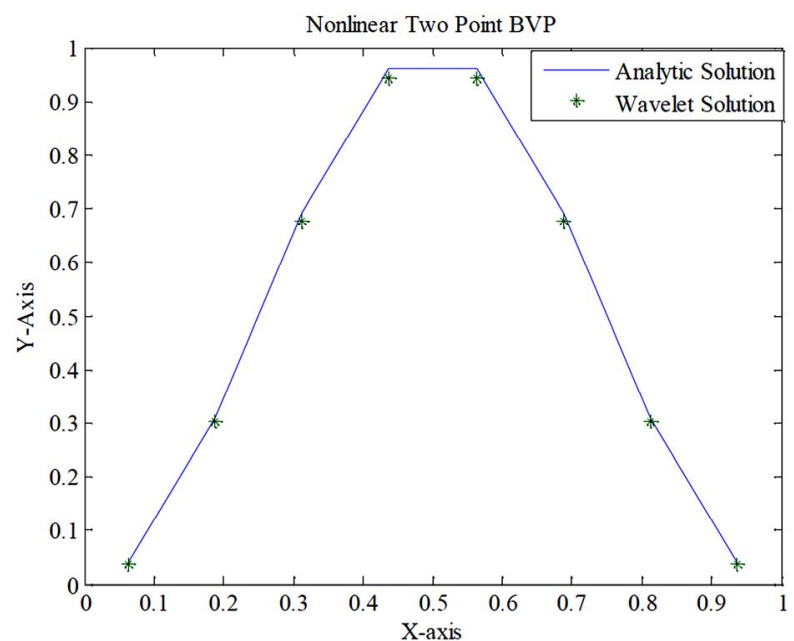

(a)

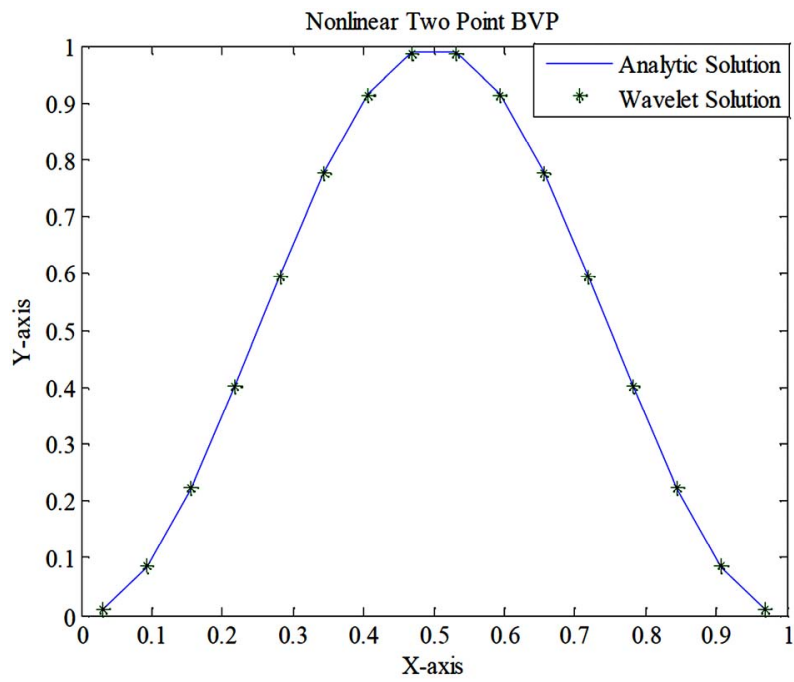

(b)

Figure 2. Comparisons of solutions. (a) For $j=2, m=4$ (b) More accurate curve when $j=3, m=8$.

$$
\begin{aligned}
y^{\prime \prime \prime}(t) & +t y(t)=t\left(1+t+\frac{1}{6} t^{3}\right) \mathrm{e}^{t}, \\
y(0) & =1, y^{\prime}(0)=1, y^{\prime \prime}(1)=1, y^{\prime \prime \prime}(1)=1, \\
y(t) & =\sum_{i=1}^{2^{j+1}} a_{i} \int_{0}^{t} \int_{0}^{t} \int_{0}^{t} \int_{0}^{t} h_{i}(t) \mathrm{d} t^{4}+\frac{t^{3}}{6}+t+1 \\
& +\left(\frac{t^{2}}{2}-\frac{t^{3}}{6}\right) \sum_{i=1}^{2^{j+1}} a_{i} \int_{0}^{1} h_{i}(t) \mathrm{d} t \\
& -\frac{t^{2}}{2} \sum_{i=1}^{2^{j+1}} a_{i} \int_{0}^{1} \int_{0}^{1} h_{i}(t) \mathrm{d} t^{2}
\end{aligned}
$$

Solution is shown in the Figure 4.

\section{Conclusions}

For nonlinear differential equations the proposed Haar

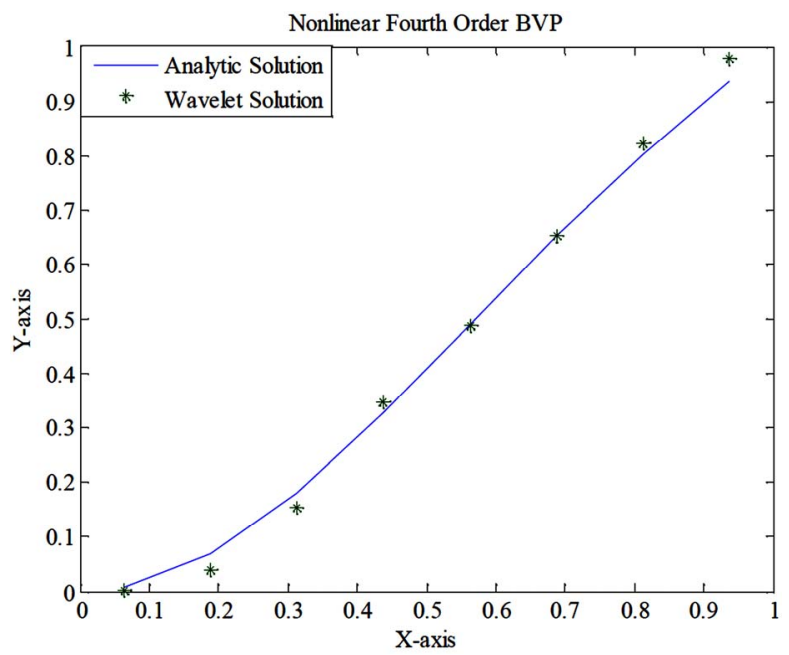

Figure 3. Comparison of Solutions for level of resolution $\boldsymbol{j}=$ $2,2 m=8$.

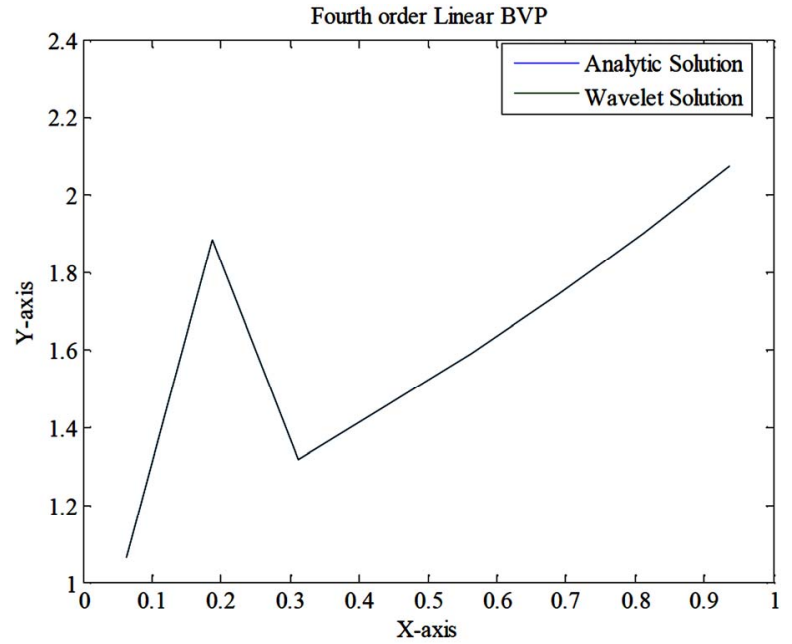

Figure 4. Comparison of Solutions for level of resolution $\boldsymbol{j}=$ $2, m=8$. 
wavelet Quasilinearization approach is adopted. The test problems of this paper demonstrate that in solving nonlinear boundary value problems the Haar wavelet method coupled with Quasilinearization approach can successfully compete with the other efficient numerical methods such as Newton-Raphson based Haar wavelet method and analytic one. The main benefits of the Haar approach are simplicity (as a small number of grid points according to the resolution guarantees the necessary accuracy without iterations) and universality (as almost the same approach is applicable for a wide class of higher order differential equations with different types of nonlinearity).

\section{References}

[1] C. F. Chen and C. H. Hsiao, "Haar Wavelet Method for Solving Lumped and Distributed-Parameter Systems," IEE Proceedings of Control Theory \& Applications, Vol. 144, No. 1, 1997, pp. 87-94. doi:10.1049/ip-cta: 19970702

[2] S. ul Islam, I. Aziz and B. Sarler, "The Numerical Solution of Second order Boundary Value Problems by Collocation Method with the Haar Wavelets," Mathematical and Computer Modelling, Vol. 52, No. 9-10, 2010, pp. 1577-1590. doi:10.1016/j.mcm.2010.06.023

[3] Z. Shi, L.-Y. Deng and Q.-J. Chen, "Numerical Solution of Differential Equations by Using Haar Wavelets," Proceedings of International Conference on Wavelet Analysis and Pattern Recognition, Beijing, 2-4 November 2007, pp. 1039-1044.

[4] U. Lepik, "Haar Wavelet Method for Solving Stiff Differential Equations," Mathematical Modelling and Analysis, Vol. 14, No. 4, 2009, pp. 467-481.

[5] J. C. Goswami and A. K. Chan, "Fundamentals of Wavelets, Theory, Algorithms and Applications," John Wiley and sons, New York, 1999.

[6] R. E. Bellman and R. E. Kalaba, "Quasilinearization and Nonlinear Boundary Value Problems," Modern Analytic and Computational Methods in Science and Mathematics, American Elsevier Publishing Company, New York, Vol. 3, 1965.

[7] V. B. Mandelzweig and F. Tabakin, "Quasilinearization Approach to Nonlinear Problems in Physics with Application to Nonlinear ODE's," Computer Physics Communication, Vol. 141, No. 2, 2001, pp. 268-281.

[8] G. Vainikko, A. Kivinukk and J. Lippus, "Fast Solvers of Integral Equations of the Second Kind: Wavelet Methods," Journal of Complexity, Vol. 21, No. 2, 2005, pp. 243-273. doi:10.1016/j.jco.2004.07.002

[9] D. S. Sophianopoulos and P. G. Asteris, "Interpolation Based Numerical Procedure for Solving Two-Point Nonlinear Boundary Value Problems," International Journal of Nonlinear Sciences and Numerical Simulation, Vol. 5, No. 1, 2006, pp. 67-78.

[10] K. N. S. K. Viswanadham, P. M. Krishna and R. S. Koneru, "Numerical Solutions of Fourth Order Boundary Value Problems by Galerkin Method with Quintic BSplines," International Journal of Nonlinear Science, Vol. 10, No. 2, 2010, pp. 222-230. 


\section{Appendix}

Here we demonstrate the developed $\mathrm{C}++$ program to solve the Haar wavelet based matrix systems for solutions of ODEs

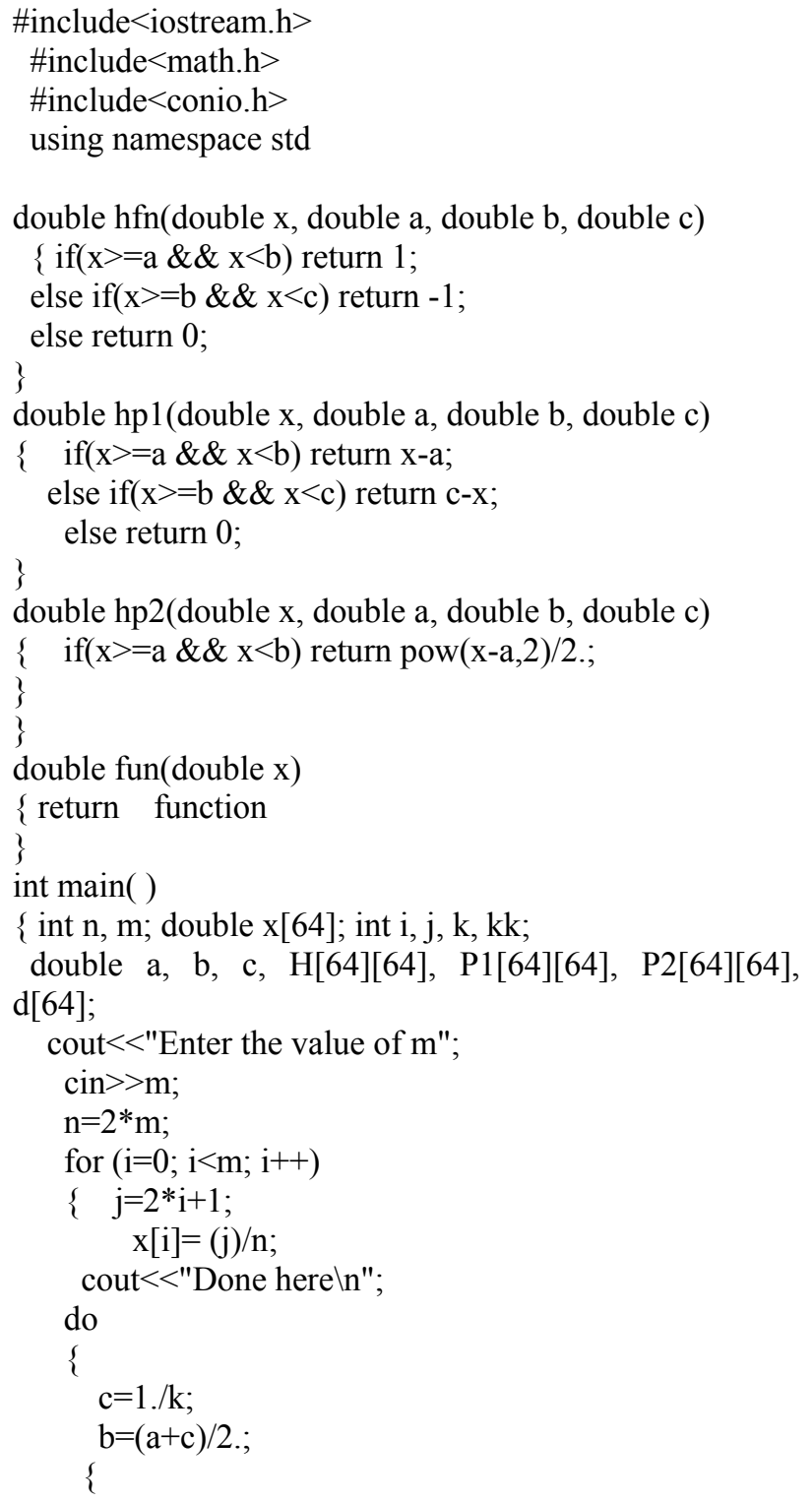

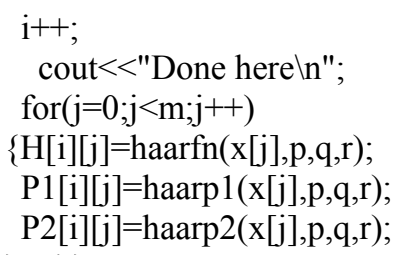

